\title{
FORCED VIBRATIONS OF STRONGLY NONLINEAR SYSTEMS WITH MULTIPLE SCALES LINDSTEDT POINCARE METHOD
}

\author{
M. Pakdemirli, M. M. F. Karahan and H. Boyac1 \\ Department of Mechanical Engineering \\ Celal Bayar University, Muradiye 45140 \\ Manisa,Turkey.mpak@bayar.edu.tr
}

\begin{abstract}
Forced vibrations of duffing equation with damping is considered. Recently developed Multiple Scales Lindstedt-Poincare (MSLP) technique for free vibrations is applied for the first time to the forced vibration problem in search of approximate solutions. For the case of weak and strong nonlinearities, approximate solutions of the new method are contrasted with the usual Multiple Scales (MS) method and numerical simulations. For weakly nonlinear systems, frequency response curves of both perturbation methods and numerical solutions are in good agreement. For strongly nonlinear systems however, results of MS deviate much from the MSLP method and numerical simulations, the latter two being in good agreement.
\end{abstract}

Keywords- Perturbation Methods, Lindstedt Poincare method, Multiple Scales method, Numerical Solutions, Forced Vibrations, Strongly Nonlinear Systems.

\section{INTRODUCTION}

Perturbation methods are well established and used for over a century to determine approximate analytical solutions for mathematical models. Algebraic equations, integrals, differential equations, difference equations and integro-differential equations can be solved approximately with these techniques. The direct expansion method (pedestrian expansion) does not produce physically valid solutions for most of the cases and depending on the nature of the equation, many different perturbation techniques such as Lindstedt-Poincare technique, Renormalization method, Method of Multiple Scales, Averaging methods, Method of Matched Asymptotic Expansions etc. are developed within time.

One of the deficiencies in applying perturbation methods is that a small parameter is needed in the equations or the small parameter should be introduced artificially to the equations. Nevertheless, the problem solved is a weak nonlinear problem and it becomes hard to obtain an approximate solution valid for strongly nonlinear systems.

There have been a number of attempts recently to validate perturbation solutions for strongly nonlinear systems also. $\mathrm{Hu}$ and Xiong [1] contrasted two different approaches of Lindstedt-Poincare methods using the duffing equation. First, they solved the equation with classical method and then they made a slight modification in the expansions. Instead of expanding the transformation frequency, they expanded the natural frequency and obtained solutions with excellent convergence properties for the duffing equation. The time histories of solutions agree with the numerical solutions for arbitrarily large perturbation parameters. In a similar paper, the approximate and exact frequencies are contrasted for the duffing equation [2]. The case of vanishing restoring force was also treated for the same equation [3]. The periods obtained are contrasted with the exact period with good convergence properties for large parameters. 
While a complete review of the attempts to validate perturbation solutions for strongly nonlinear oscillators is beyond the scope of this work, a partial list will be given. Among the many developed methods, Linearized perturbation method [4-6], parameter expanding method [7,8], new time transformations as modifications of Lindstedt-Poincare method [9-11], iteration methods $[12,14]$ are some examples.

Very recently, Pakdemirli et al. [15] proposed a new perturbation method to handle strongly nonlinear systems. The method combines Multiple Scales and Lindstedt Poincare method with a frequency expansion suggested in references [1,2]. The justification for combining both methods is that Multiple Scales is better in determining transient solutions while Lindstedt Poincare method may be better under some circumstances in determining steady state solutions [16]. The new method, namely the Multiple Scales Lindstedt Poincare method (MSLP), is applied to free vibrations of a linear damped oscillator, undamped and damped duffing oscillator. It is shown that exact analytical solution can be retrieved by the new method for the linear damped oscillator. For undamped and damped duffing oscillators, results of the new method are in good agreement with the numerical simulations for strong nonlinearities.

This recently developed method is applied to an equation with quadratic and cubic nonlinearities [17]. Approximate analytical solutions are found using the classical Multiple Scales method and the new method. Both solutions are contrasted with the direct numerical solutions of the original equation. For the case of strong nonlinearities, solutions of the new method are in good agreement with the numerical results whereas amplitude and frequency estimations of classical Multiple Scales yield high errors. For strongly nonlinear systems, exact periods match well with the new technique while there are large discrepancies between the exact and classical Multiple Scale periods.

In this work, MSLP method is applied for the first time to a forced vibration problem. Primary resonances are considered in this study. The expansions of natural and external frequencies to obtain valid solutions are nontrivial and the outline of the method is given for the forced vibrations of a duffing equation with damping. Frequency response curves of MS, MSLP are contrasted with direct numerical simulations. While all three methods are in reasonable agreement for weakly nonlinear systems, for strong nonlinearities, unphysical extraneous solutions appear in MS. Results of MSLP and numerical solutions are consistent with each other however.

\section{MULTIPLE SCALES (MS) METHOD}

In this section, the forced vibrations of the damped duffing oscillator

$$
\ddot{\mathrm{u}}+\omega_{0}{ }^{2} \mathrm{u}+2 \varepsilon^{2} \mu \dot{\mathrm{u}}+\varepsilon \alpha \mathrm{u}^{3}=\varepsilon^{2} \mathrm{f} \cos \Omega \mathrm{t}
$$

will be treated with the usual Multiple Scales [18] method. A higher order perturbation method to see the effect of correction term openly is preferred and external excitation and damping are re-ordered to appear at the last order of approximation. Fast and slow time scales

$$
\mathrm{T}_{0}=\mathrm{t}, \quad \mathrm{T}_{1}=\varepsilon \mathrm{t}, \quad \mathrm{T}_{2}=\varepsilon^{2} \mathrm{t}
$$

and the time derivatives with respect to these variables are

$$
\frac{\mathrm{d}}{\mathrm{dt}}=\mathrm{D}_{0}+\varepsilon \mathrm{D}_{1}+\varepsilon^{2} \mathrm{D}_{2}+\ldots, \quad \frac{\mathrm{d}^{2}}{\mathrm{dt}^{2}}=\mathrm{D}_{0}^{2}+2 \varepsilon \mathrm{D}_{0} \mathrm{D}_{1}+\varepsilon^{2}\left(\mathrm{D}_{1}^{2}+2 \mathrm{D}_{0} \mathrm{D}_{2}\right)+\ldots
$$


The approximate expansion

$$
\mathrm{u}=\mathrm{u}_{0}\left(\mathrm{~T}_{0}, \mathrm{~T}_{1}, \mathrm{~T}_{2}\right)+\varepsilon \mathrm{u}_{1}\left(\mathrm{~T}_{0}, \mathrm{~T}_{1}, \mathrm{~T}_{2}\right)+\varepsilon^{2} \mathrm{u}_{2}\left(\mathrm{~T}_{0}, \mathrm{~T}_{1}, \mathrm{~T}_{2}\right)+\ldots
$$

is inserted into (1) and separated

$\mathrm{O}(1): \mathrm{D}_{0}^{2} \mathrm{u}_{0}+\omega_{0}^{2} \mathrm{u}_{0}=0$

$\mathrm{O}(\varepsilon): \mathrm{D}_{0}^{2} \mathrm{u}_{1}+\omega_{0}^{2} \mathrm{u}_{1}=-2 \mathrm{D}_{0} \mathrm{D}_{1} \mathrm{u}_{0}-\alpha \mathrm{u}_{0}^{3}$

$\mathrm{O}\left(\varepsilon^{2}\right): \mathrm{D}_{0}^{2} \mathrm{u}_{2}+\omega_{0}^{2} \mathrm{u}_{2}=-2 \mathrm{D}_{0} \mathrm{D}_{1} \mathrm{u}_{1}-\left(\mathrm{D}_{1}^{2}+2 \mathrm{D}_{0} \mathrm{D}_{2}\right) \mathrm{u}_{0}-2 \mu \mathrm{D}_{0} \mathrm{u}_{0}-3 \alpha \mathrm{u}_{0}^{2} \mathrm{u}_{1}+\mathrm{f} \cos \Omega \mathrm{T}_{0}$

The solution at the first order

$$
\mathrm{u}_{0}=\mathrm{A}\left(\mathrm{T}_{1}, \mathrm{~T}_{2}\right) \mathrm{e}^{\mathrm{i} \omega_{0} \mathrm{~T}_{0}}+\mathrm{cc}
$$

is inserted into the right hand side of $\mathrm{O}(\varepsilon)$ equation and elimination of secularities yield

$$
2 i \omega_{0} D_{1} A=-3 \alpha A^{2} \bar{A}
$$

Note that cc stands for complex conjugates of the preceding terms. Substitution of the polar form

$$
\mathrm{A}=\frac{1}{2} \mathrm{ae}^{\mathrm{i} \beta}
$$

and separation of real and imaginary parts results in

$$
\mathrm{a}=\mathrm{a}\left(\mathrm{T}_{2}\right), \quad \beta=\frac{3 \alpha}{8 \omega_{0}} \mathrm{a}^{2} \mathrm{~T}_{1}+\beta_{0}\left(\mathrm{~T}_{2}\right)
$$

The solution at this order is

$\mathrm{u}_{1}=\frac{\alpha}{8 \omega_{0}^{2}} \mathrm{~A}^{3} \mathrm{e}^{3 \mathrm{i} \omega_{0} \mathrm{~T}_{0}}+\mathrm{cc}=\frac{\alpha}{32 \omega_{0}^{2}} \mathrm{a}^{3} \cos \left(3 \omega_{0} \mathrm{~T}_{0}+3 \beta\right)$

Inserting (12) and (8) to $\mathrm{O}\left(\varepsilon^{2}\right)$ equation, assuming

$$
\Omega=\omega_{0}+\varepsilon^{2} \sigma
$$

for primary resonances and eliminating secular terms yield finally

$$
2 i \omega_{0} D_{2} A+D_{1}^{2} A+2 \mu i \omega_{0} A+\frac{3 \alpha^{2}}{8 \omega_{0}{ }^{2}} A^{3} \bar{A}^{2}-\frac{f}{2} e^{i \sigma T_{2}}=0
$$

For determining amplitude and phase modulations, for higher order solutions, the usual reconstitution method [19] will be employed. Hence, $D_{1} A$ is inserted from (9) to determine $\mathrm{D}_{2} \mathrm{~A}$

$$
2 i \omega_{0} D_{2} A=\frac{15 \alpha^{2}}{8 \omega_{0}{ }^{2}} A^{3} \bar{A}^{2}-2 \mu i \omega_{0} A+\frac{f}{2} e^{i \sigma T_{2}}
$$

The complex amplitude modulations are

$$
2 \mathrm{i} \omega_{0} \frac{\mathrm{dA}}{\mathrm{dt}}=2 \mathrm{i} \omega_{0}\left(\varepsilon \mathrm{D}_{1} \mathrm{~A}+\varepsilon^{2} \mathrm{D}_{2} \mathrm{~A}\right)
$$

or

$$
2 i \omega_{0} \frac{d A}{d t}=\varepsilon\left(-3 \alpha A^{2} \bar{A}\right)+\varepsilon^{2}\left(\frac{15 \alpha^{2}}{8 \omega_{0}{ }^{2}} A^{3} \bar{A}^{2}-2 \mu i \omega_{0} A+\frac{f}{2} e^{i \sigma T_{2}}\right)
$$

Insertion of the polar form, separation into real and imaginary parts finally yield the amplitude and phase modulation equations 


$$
\begin{aligned}
& \dot{\mathrm{a}}=\varepsilon^{2}\left(-\mu \mathrm{a}+\frac{\mathrm{f}}{2 \omega_{0}} \sin \gamma\right) \\
& \dot{\gamma}=-\frac{3 \varepsilon \alpha}{8 \omega_{0}} \mathrm{a}^{2}+\varepsilon^{2}\left(\sigma+\frac{15 \alpha^{2}}{256 \omega_{0}{ }^{3}} \mathrm{a}^{4}+\frac{\mathrm{f}}{2 \mathrm{a} \omega_{0}} \cos \gamma\right)
\end{aligned}
$$

where dot denotes differentiation with respect to time $t$ and phase $\gamma$ is defined to be

$$
\gamma=\sigma \mathrm{T}_{2}-\beta
$$

For steady state solutions, $\dot{\mathrm{a}}=\dot{\gamma}=0$ in (18) and (19). Frequency detuning parameter $\sigma$ can be calculated by elimination of $\gamma$, which upon substitution into (13) yields the frequency response relation

$$
\Omega=\omega_{0}+\varepsilon\left(\frac{3 \alpha}{8 \omega_{0}} \mathrm{a}^{2}\right)+\varepsilon^{2}\left(-\frac{15 \alpha^{2}}{256 \omega_{0}{ }^{3}} \mathrm{a}^{4} \pm \sqrt{\frac{\mathrm{f}^{2}}{4 \mathrm{a}^{2} \omega_{0}{ }^{2}}-\mu^{2}}\right)
$$

The approximate solution is

$$
\mathrm{u}=\mathrm{a} \cos (\Omega \mathrm{t}-\gamma)+\varepsilon \frac{\alpha \mathrm{a}^{3}}{32 \omega_{0}{ }^{2}} \cos [3(\Omega \mathrm{t}-\gamma)]+\mathrm{O}\left(\varepsilon^{2}\right)
$$

where real amplitudes a and phases $\gamma$ are governed by (18) and (19).

\section{MULTIPLE SCALES LINDSTEDT POINCARE (MSLP) METHOD}

Recently developed Multiple Scales Lindstedt Poincare method [15] will be applied to the forced vibrations for the first time to obtain approximate expansions.

The time transformation

$$
\tau=\omega \mathrm{t}
$$

is applied to (1)

$$
\omega^{2} u^{\prime \prime}+\omega_{0}^{2} u+2 \varepsilon^{2} \mu \omega u^{\prime}+\varepsilon \alpha u^{3}=\varepsilon^{2} f \cos \frac{\Omega}{\omega} T_{0}
$$

Note that a time transformation involving $\tau=\Omega$ t instead of $\omega$ would not be appropriate.

Prime represents derivative with respect to time variable $\tau$. Fast and slow time scales are

Using

$$
\mathrm{T}_{0}=\tau, \quad \mathrm{T}_{1}=\varepsilon \tau, \quad \mathrm{T}_{2}=\varepsilon^{2} \tau
$$

$$
\frac{\mathrm{d}^{2}}{\mathrm{~d} \tau^{2}}=\mathrm{D}_{0}^{2}+2 \varepsilon \mathrm{D}_{0} \mathrm{D}_{1}+\varepsilon^{2}\left(\mathrm{D}_{1}^{2}+2 \mathrm{D}_{0} \mathrm{D}_{2}\right)+\ldots
$$

where $\mathrm{D}_{\mathrm{n}}=\partial / \partial \mathrm{T}_{\mathrm{n}}$ and substituting the expansions

$$
\begin{aligned}
& \mathrm{u}=\mathrm{u}_{0}\left(\mathrm{~T}_{0}, \mathrm{~T}_{1}, \mathrm{~T}_{2}\right)+\varepsilon \mathrm{u}_{1}\left(\mathrm{~T}_{0}, \mathrm{~T}_{1}, \mathrm{~T}_{2}\right)+\varepsilon^{2} \mathrm{u}_{2}\left(\mathrm{~T}_{0}, \mathrm{~T}_{1}, \mathrm{~T}_{2}\right)+\ldots \\
& \omega_{0}^{2}=\omega^{2}-\varepsilon \omega_{1}-\varepsilon^{2} \omega_{2}
\end{aligned}
$$

into (24) yields after separation

$\mathrm{O}(1): \omega^{2} \mathrm{D}_{0}^{2} \mathrm{u}_{0}+\omega^{2} \mathrm{u}_{0}=0$

$\mathrm{O}(\varepsilon): \omega^{2} \mathrm{D}_{0}^{2} \mathrm{u}_{1}+\omega^{2} \mathrm{u}_{1}=-2 \omega^{2} \mathrm{D}_{0} \mathrm{D}_{1} \mathrm{u}_{0}+\omega_{1} \mathrm{u}_{0}-\alpha \mathrm{u}_{0}^{3}$ 


$$
\mathrm{O}\left(\varepsilon^{2}\right): \begin{array}{r}
\omega^{2} \mathrm{D}_{0}^{2} \mathrm{u}_{2}+\omega^{2} \mathrm{u}_{2}=-2 \omega^{2} \mathrm{D}_{0} \mathrm{D}_{1} \mathrm{u}_{1}-\omega^{2}\left(\mathrm{D}_{1}^{2}+2 \mathrm{D}_{0} \mathrm{D}_{2}\right) \mathrm{u}_{0}+\omega_{1} \mathrm{u}_{1} \\
+\omega_{2} \mathrm{u}_{0}-2 \mu \omega \mathrm{D}_{0} \mathrm{u}_{0}-3 \alpha \mathrm{u}_{0}^{2} \mathrm{u}_{1}+\mathrm{f} \cos \frac{\Omega}{\omega} \mathrm{T}_{0}
\end{array}
$$

Note that following [1,2], in equation (28), instead of transformation frequency, the natural frequency is expanded. The solution at the first order is

$$
\mathrm{u}_{0}=\mathrm{Ae}^{\mathrm{i \textrm {T } _ { 0 }}}+\mathrm{cc}=\mathrm{a} \cos \left(\mathrm{T}_{0}+\beta\right)
$$

This solution is substituted into the right hand side of (30) and secular terms are eliminated

$$
-2 \mathrm{i} \omega^{2} \mathrm{D}_{1} \mathrm{~A}+\omega_{1} \mathrm{~A}-3 \alpha \mathrm{A}^{2} \overline{\mathrm{A}}=0
$$

In MSLP as outlined in [15], first $D_{1} A=0$ is selected and if the frequency correction is real, this choice is admissible. If $\omega_{1}$ turns out to be complex, then $D_{1} A \neq 0$ which implies $\omega_{1}=0$ and secularities are eliminated by choosing $D_{1} A$. A complex $\omega_{1}$ implies that there is amplitude variation and LP method fails to produce physical solutions [18]. The method allows switching back and forth with MS and LP type of eliminating secularities thereby augmenting the advantages of both methods. For equation (33), selection of

$$
\mathrm{D}_{1} \mathrm{~A}=0 \Rightarrow \mathrm{A}=\mathrm{A}\left(\mathrm{T}_{2}\right)
$$

produces

$$
\omega_{1}=3 \alpha \mathrm{A} \overline{\mathrm{A}}=\frac{3}{4} \alpha \mathrm{a}^{2}
$$

which is suitable because $\omega_{1}$ is real. The solution at order $\varepsilon$ is

$$
\mathrm{u}_{1}=\frac{\alpha}{8 \omega^{2}} \mathrm{~A}^{3} \mathrm{e}^{3 \mathrm{i} \mathrm{T}_{0}}+\mathrm{cc}=\frac{\alpha}{32 \omega^{2}} \mathrm{a}^{3} \cos \left(3 \mathrm{~T}_{0}+3 \beta\right)
$$

For the last order of approximation, the nearness of excitation frequency to the transformation frequency is expressed as follows

$$
\Omega=\omega\left(1+\varepsilon^{2} \sigma\right)
$$

Substitution of (32), (36) and (37) into the right hand side of (31) and elimination of secularities yield

$$
-2 i \omega^{2} D_{2} A+\omega_{2} A-2 \mu i \omega A-\frac{3 \alpha^{2}}{8 \omega^{2}} A^{3} \bar{A}^{2}+\frac{f}{2} e^{i \sigma T_{2}}=0
$$

$\mathrm{D}_{2} \mathrm{~A}$ cannot be selected as zero, since $\omega_{2}$ would then be complex. Therefore the admissible choice is

$$
\omega_{2}=0
$$

and

$$
-2 i \omega^{2} D_{2} A-2 \mu i \omega A-\frac{3 \alpha^{2}}{8 \omega^{2}} A^{3} \bar{A}^{2}+\frac{f}{2} e^{i \sigma T_{2}}=0
$$

The polar form $\mathrm{A}=\frac{1}{2} \mathrm{ae}^{\mathrm{i} \beta}$ is substituted, real and imaginary parts are separated

$\mathrm{D}_{2} \mathrm{a}=-\frac{\mu}{\omega} \mathrm{a}+\frac{\mathrm{f}}{2 \omega^{2}} \sin \gamma$ 
$\mathrm{D}_{2} \gamma=\sigma-\frac{3 \alpha^{2}}{256 \omega^{4}} \mathrm{a}^{4}+\frac{\mathrm{f}}{2 \mathrm{a} \omega^{2}} \cos \gamma$

where

$$
\gamma=\sigma \mathrm{T}_{2}-\beta
$$

For steady state solutions, $\mathrm{D}_{2} \mathrm{a}=0, \mathrm{D}_{2} \gamma=0$ and elimination of $\gamma$ between (41) and (42) yields

$$
\sigma=\frac{3 \alpha^{2}}{256 \omega^{4}} a^{4} \pm \sqrt{\frac{\mathrm{f}^{2}}{4 \mathrm{a}^{2} \omega^{4}}-\left(\frac{\mu}{\omega}\right)^{2}}
$$

From (37), frequency-response relation is

$\Omega=\omega\left[1+\varepsilon^{2}\left(\frac{3 \alpha^{2}}{256 \omega^{4}} a^{4} \pm \sqrt{\frac{\mathrm{f}^{2}}{4 \mathrm{a}^{2} \omega^{4}}-\left(\frac{\mu}{\omega}\right)^{2}}\right)\right]$

where

$$
\omega=\sqrt{\omega_{0}^{2}+\varepsilon \frac{3}{4} \alpha a^{2}}
$$

Equation (46) is obtained by substituting (39) and (35) into (28). The approximate solution is

$$
u=a \cos (\Omega t-\gamma)+\varepsilon \frac{\alpha}{32 \omega^{2}} a^{3} \cos [3(\Omega t-\gamma)]+O\left(\varepsilon^{2}\right)
$$

The amplitude and phases are governed by

$$
\begin{aligned}
& \dot{\mathrm{a}}=\varepsilon^{2}\left(-\mu \mathrm{a}+\frac{\mathrm{f}}{2 \omega} \sin \gamma\right) \\
& \dot{\gamma}=\varepsilon^{2}\left(\omega \sigma-\frac{3 \alpha^{2}}{256 \omega^{3}} \mathrm{a}^{4}+\frac{\mathrm{f}}{2 \mathrm{a} \omega} \cos \gamma\right)
\end{aligned}
$$

\section{COMPARISONS WITH THE NUMERICAL SOLUTIONS}

Frequency response relations obtained by both methods will be contrasted by direct numerical integrations of the equation. For perturbation solutions to be valid, the correction term should be much smaller than the leading term. For both methods, the requirements are

$$
\begin{aligned}
& \frac{\varepsilon \alpha \mathrm{a}^{2}}{32 \omega_{0}^{2}}<<1 \quad \text { (MS) } \\
& \frac{\varepsilon \alpha \mathrm{a}^{2}}{32 \omega^{2}}<<1 \quad \text { (MSLP) }
\end{aligned}
$$

The only difference in the criteria is the replacement of $\omega$ instead of $\omega_{0}$ in the MSLP method. For strong nonlinearities, $\alpha$ should be arbitrarily large. For MS, taking the limit $\lim _{\alpha \rightarrow \infty} \frac{\varepsilon \alpha}{32 \omega_{0}^{2}} \mathrm{a}^{2}=\infty$ 
yields infinity as expected. Hence MS solution can not be valid for large values of $\alpha$. In contrast, for MSLP, the corresponding limit is

$$
\lim _{\alpha \rightarrow \infty} \frac{\varepsilon \alpha}{32 \omega^{2}} \mathrm{a}^{2}=\frac{\varepsilon \alpha \mathrm{a}^{2}}{32\left(\omega_{0}^{2}+\varepsilon \frac{3 \alpha}{4} \mathrm{a}^{2}\right)}=\frac{1}{24}<<1
$$

which satisfies the perturbation requirement for arbitrarily large nonlinearities.

Frequency response relations are contrasted with the numerical simulations as a next step. In Figure 1, all parameters are selected within the preordered range with a weak nonlinearity $(\alpha=1)$. As expected, frequency response curves of both methods coincide. The numerical simulation results are obtained by integrating the original equation (1) and determining the steady state amplitudes by taking a sufficient interval of time history. Numerical results are labeled by dots on the graphs. Numerical simulations also confirm the frequency response curves of both methods. In Figure 2, the cubic nonlinearity is increased substantially $(\alpha=100)$. Extra unphysical solution branches appear for MS which cannot be verified with the numerical simulations. In contrast, MSLP solutions are in good agreement with the numerical ones. Taking $\alpha=1000$ in Figure 3 still shows the same trend, that is MS solutions being quantitatively and qualitatively much different than the others with MSLP and numerical simulations being in good agreement. If the nonlinearity is not large $(\alpha=1)$, but the external excitation amplitude is increased $(f=10)$ with a smaller damping coefficient $(\mu=0.2)$ as in Figure 4, MS predicts a wider range for jump phenomena which can not be verified by numerical simulations. Finally if the external excitation is further increased $(f=20)$, for MS, the unphysical solutions of backward curving re-appears similar to the case of strong nonlinearities. However, MSLP stands in good agreement with the numerical simulations.

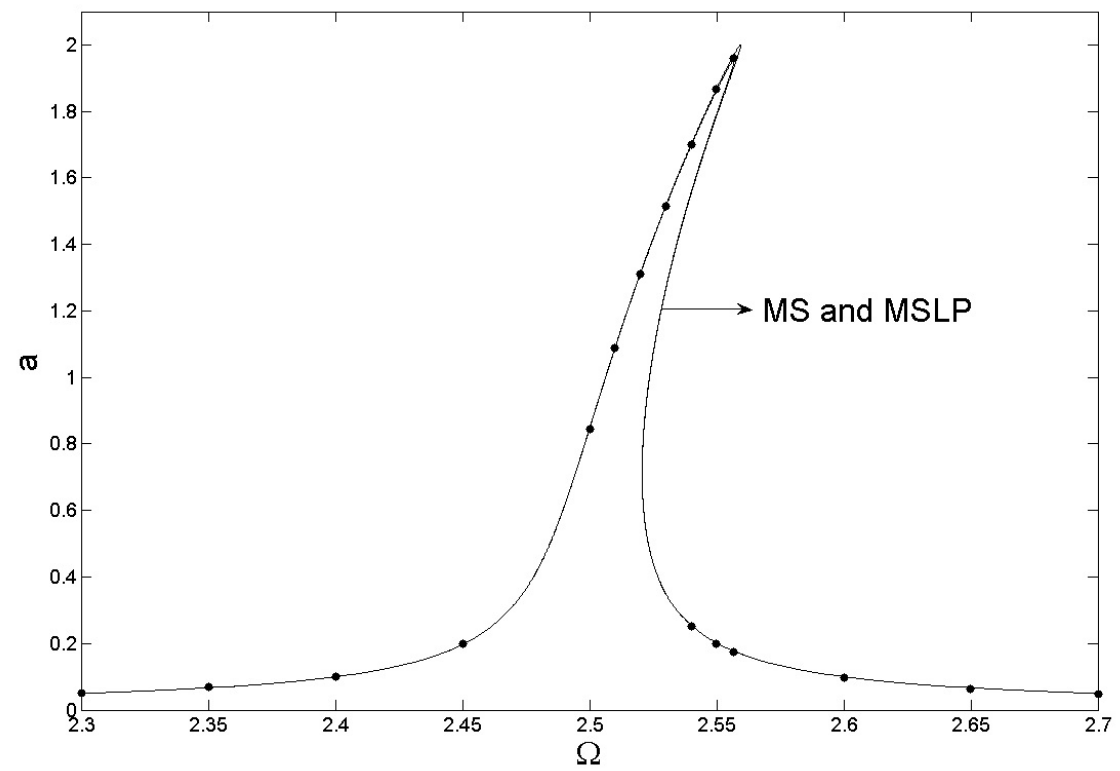

Figure 1- Comparison of frequency response curves of the MS, MSLP Method and Numerical Simulations (Represented by dots) $\left(\varepsilon=0.1, \alpha=1, \mathrm{f}=5, \omega_{0}=2.5, \mu=0.5\right)$ 


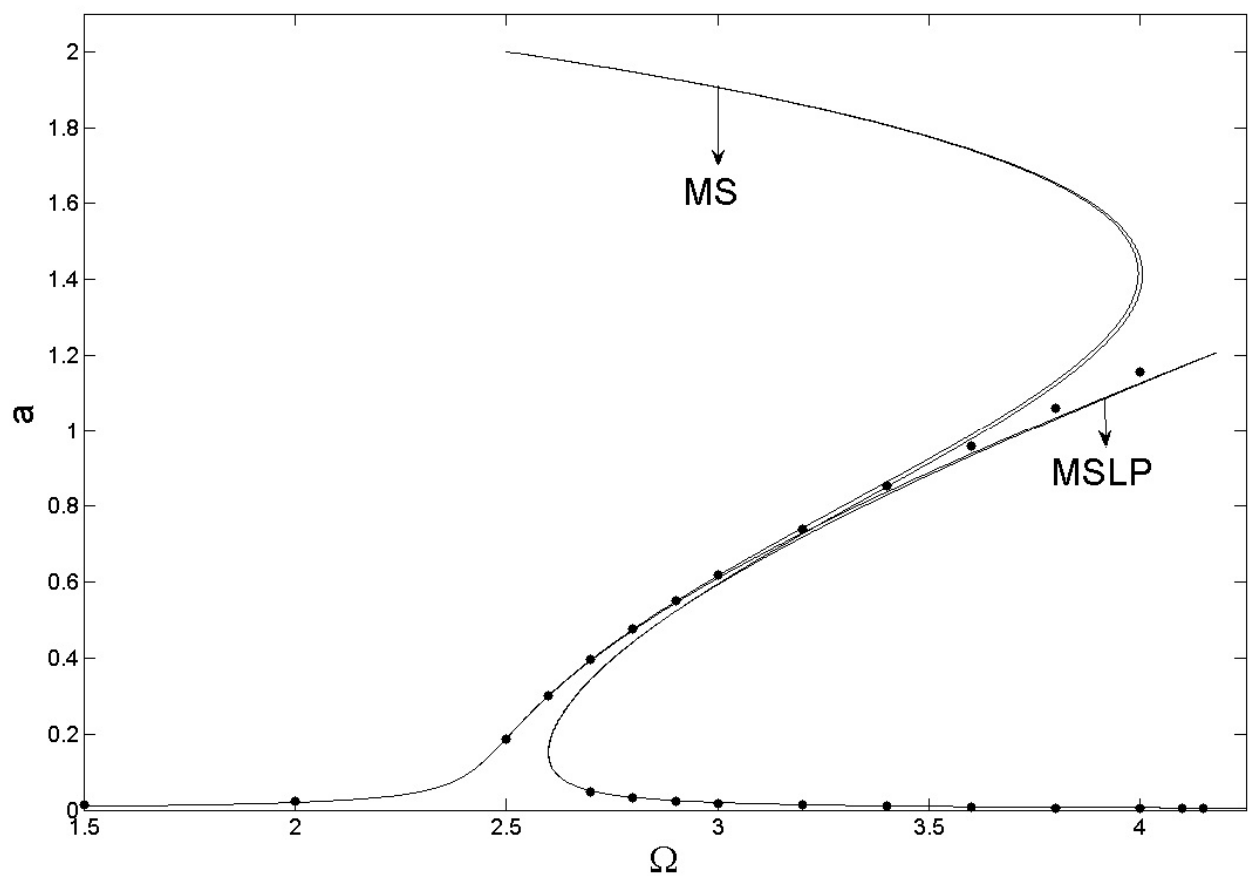

Figure 2- Comparison of frequency response curves of the MS, MSLP Method and Numerical Simulations (Represented by dots) $\left(\varepsilon=0.1, \alpha=100, \mathrm{f}=5, \omega_{0}=2.5, \mu=0.5\right)$

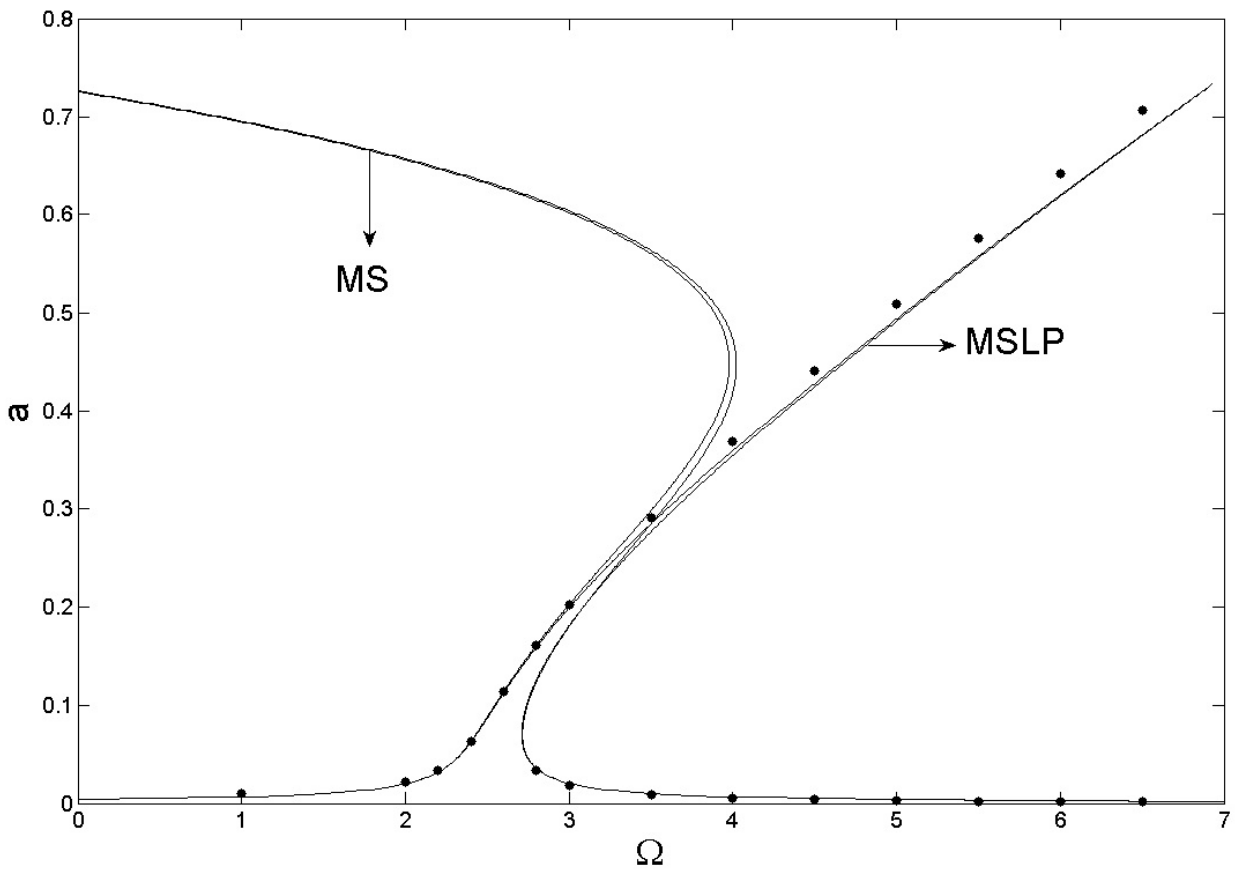

Figure 3- Comparison of frequency response curves of the MS, MSLP Method and Numerical Simulations (Represented by dots) $\left(\varepsilon=0.1, \alpha=1000, f=5, \omega_{0}=2.5, \mu=0.5\right)$ 


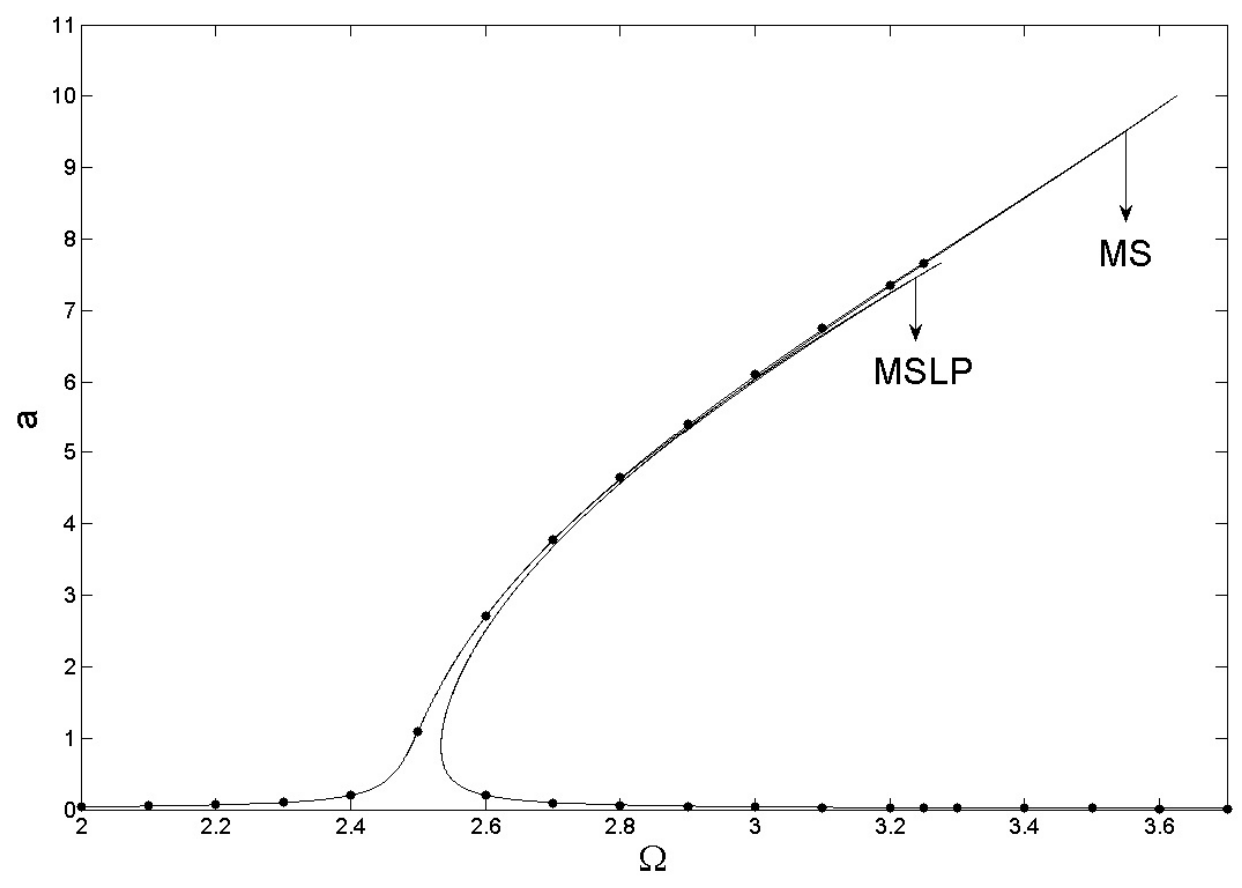

Figure 4- Comparison of frequency response curves of the MS, MSLP Method and Numerical Simulations (Represented by dots) $\left(\varepsilon=0.1, \alpha=1, f=10, \omega_{0}=2.5, \mu=0.2\right)$

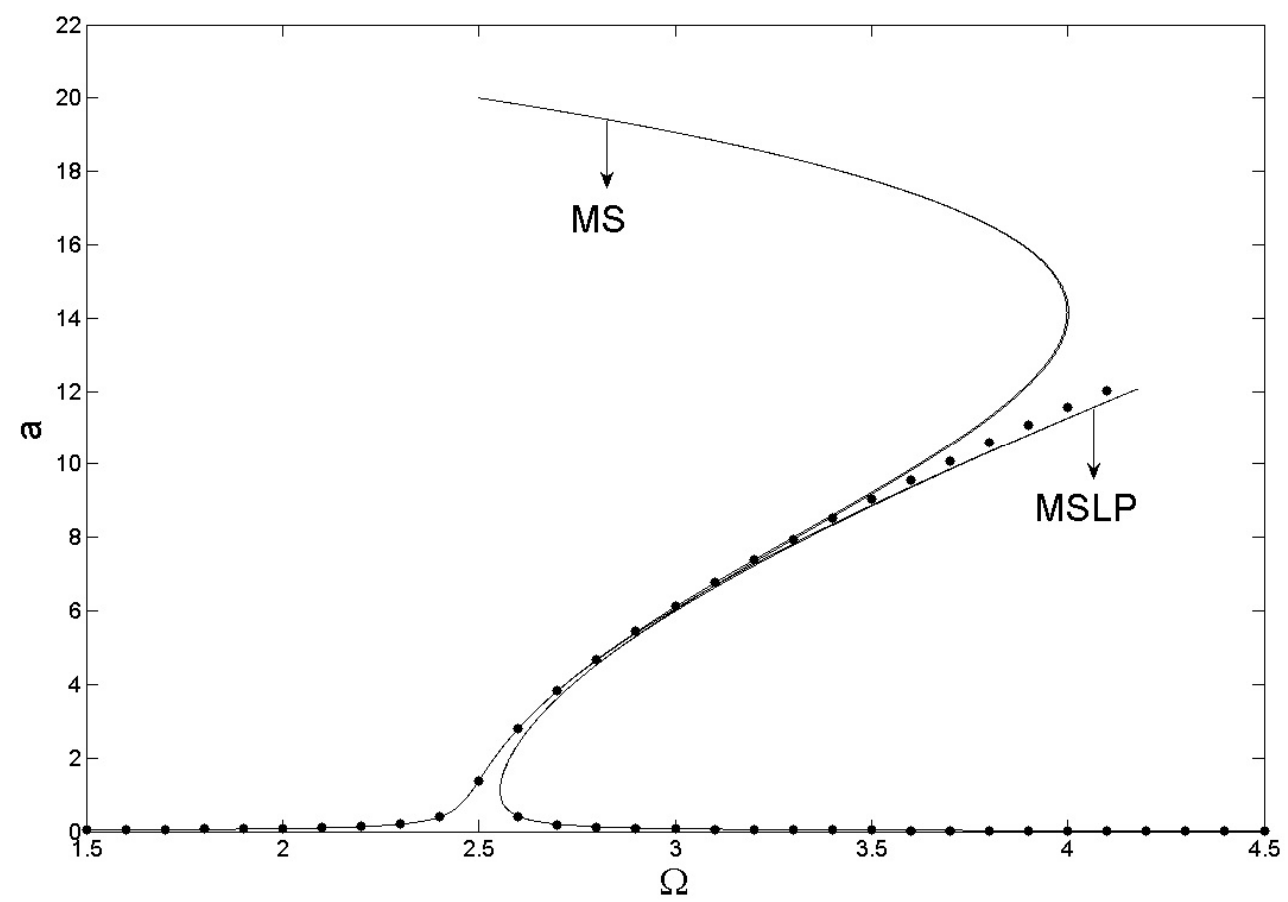

Figure 5- Comparison of frequency response curves of the MS, MSLP Method and Numerical Simulations (Represented by dots) $\left(\varepsilon=0.1, \alpha=1, f=20, \omega_{0}=2.5, \mu=0.2\right)$ 


\section{CONCLUDING REMARKS}

The new perturbation technique combining the Multiple Scales and Lindstedt Poincare method developed in [15] is applied to forced vibrations for the first time. Primary resonances are considered in the study and frequency expansions are outlined for the new method. Approximate solutions and frequency response curves are derived for the usual Multiple Scales and the new Multiple Scales Lindstedt Poincare method. To test the solutions, direct numerical integrations of the equations are done. The following conclusions can be drawn from the study

1) The new MSLP method can be successfully applied to forced vibration problems with strong nonlinearities also.

2) For weak nonlinearities and/or other parameters selected without order violations, MS, MSLP and numerical solutions are in good agreement.

3) For strong nonlinearities and/or other parameters selected with order violations, unphysical backward curves appear in MS while numerical and MSLP solutions still agree well.

4) MSLP definitely improves the classical and well established MS solutions to have a wider range of validity.

A further study would be to apply this new technique to partial differential equations. The nonlinearities arising in partial differential equations are classified using a suitable operator notation and general solution algorithms were developed for the models previously [20-22].

Acknowledgment- This work is supported by The Scientific and Technological Research Council of Turkey (TUBİTAK) under project number 108M490.

\section{REFERENCES}

1. H. Hu and Z. G. Xiong, Comparison of two Lindstedt-Poincare type perturbation methods, Journal of Sound and Vibration 278: 437-444 (2004).

2. $\mathrm{H}$. Hu, A classical perturbation technique which is valid for large parameters, Journal of Sound and Vibration 269: 409-412 (2004).

3. H. Hu, A classical perturbation technique that works even when the linear part of restoring force is zero, Journal of Sound and Vibration 271: 1175-1179 (2004).

4. J. H. He, Linearized perturbation technique and its applications to strongly nonlinear oscillators, Computers and Mathematics with Applications 45: 1-8 (2003).

5. H. Hu, A modified method of equivalent linearization that works even when the nonlinearity is not small, Journal of Sound and Vibration 276: 1145-1149 (2004).

6. J. H. He, Modified straightforward expansion, Meccanica 34: 287-289 (1999).

7. J. H. He, Some asymptotic methods for strongly nonlinear equations, International Journal of Modern Physics B 20: 1141-1199 (2006).

8. L. $\mathrm{Xu}$, Determination of limit cycle by He's parameter-expanding method for strongly nonlinear oscillators, Journal of Sound and Vibration 302: 178-184 (2007).

9. S. Q. Dai, On a generalized PLK method and its applications, Acta Mechanica Sinica 6: 111-118 (1990). 
10. J. H. He, Modified Lindstedt-Poincare methods for some strongly nonlinear oscillations Part I: expansion of a constant, International Journal of Non-Linear Mechanics 37: 309-314 (2002).

11. J. H. He, Modified Lindstedt-Poincare methods for some strongly nonlinear oscillations Part II: a new transformation, International Journal of Non-Linear Mechanics 37: 315-320 (2002).

12. V. Marinca and N. Herisanu, A modified iteration perturbation method for some nonlinear oscillation problems, Acta Mechanica 184: 231-242 (2006).

13. $\mathrm{H}$. $\mathrm{Hu}$, Solutions of a quadratic nonlinear oscillator: Iteration procedure, Journal of Sound and Vibration 298: 1159-1165 (2006).

14.Yiğit Aksoy, Mehmet Pakdemirli, New perturbation iteration solutions for Bratutype equations, Computers \& Mathematics with Applications, Volume 59, Issue 8, 2802-2808, 2010

15. M. Pakdemirli, M. M. F. Karahan and H. Boyac1, A New Perturbation Algorithm with Better Convergence Properties: Multiple Scales Lindstedt Poincare Method, Mathematical and Computational Applications 14: 31-44 (2009).

16. M. Pakdemirli, Comparison of higher order versions of the method of multiple scales for an odd nonlinearity problem, Journal of Sound and Vibration 262: 989-998 (2003).

17. M. Pakdemirli and M. M. F. Karahan, A New Perturbation Solution for Systems with Strong Quadratic and Cubic Nonlinearities, Mathematical Methods in the Applied Sciences 33, 704-712, 2010

18. A. H. Nayfeh, Introduction to Perturbation Techniques, John Wiley and Sons, New York 1981.

19. A. H. Nayfeh, Resolving controversies in the application of the method of Multiple Scales and the Generalized Method of Averaging, Nonlinear Dynamics 40: 61-102, (2005).

20. M. Pakdemirli, A comparison of two perturbation methods for vibrations of systems with quadratic and cubic nonlinearities, Mechanics Research Communications 21: 203208, (1994).

21. M. Pakdemirli and H. Boyaci, Comparison of direct-perturbation methods with discretization-perturbation methods for nonlinear vibrations, Journal of Sound and Vibration 186: 837-845, (1995).

22. M. Pakdemirli, Vibrations of continuous systems with a general operator notation suitable for perturbative calculations, Journal of Sound and Vibration 246(5), 841-851, 2001. 